\title{
THEORETICAL BACKGROUND OF INTELLECTUAL DISABILITY: POSSIBILITIES AND LIMITATIONS
}

\author{
Mariia Malaniiuk \\ Senior lector, Lviv State University of Life Safety, \\ e-mail: maria.malanyuk@gmail.com, orcid.org/0000-0002-1290-0407, Ukraine
}

\begin{abstract}
The article is focused on the problem of disability as a general mental disease and mental retardation as a kind of intellectual disorder and possible alternative ways of teaching English as a foreign language. The authors define a mental disorder, also called a mental illness or psychiatric disorder, as a behavioral or mental pattern that causes significant distress or impairment of personal functioning. They claim that abilities and limitations of mentally handicapped children are presented by defining mental retardation. The article also presents the classification of mental disabilities, and a concrete type of the mental disability the mild one is characterized. It also substantiates the objective necessity that a skilled teacher should be very flexible and methodologically well prepared for teaching English as a foreign language to mentally handicapped students, using different methods tailored to their individual needs.
\end{abstract}

Keywords: intellectual disorder, mild mental disorder, mentally handicapped students teaching methods.

\section{DOI: http://dx.doi.org/10.23856/3612}

\section{Introduction}

A mental disorder, also called a mental illness or psychiatric disorder, is a behavioral or mental pattern that causes significant distress or impairment of personal functioning. Such features may be persistent, relapsing and remitting, or occur as a single episode. Many disorders have been described, with signs and symptoms that vary widely between specific disorders. Such disorders may be diagnosed by a mental health professional.

According to WHO there are about 1000000000 people with different kind of disability. It is $15 \%$ of the whole world population (World Health Organization). In a modern, highly developed world, intellectual disability affects about $3 \%$ of it. The 2004 WHO report "Prevention of Mental Disorders" stated that "Prevention of these disorders is obviously one of the most effective ways to reduce the [disease] burden" (World Health Organization). The 2011 European Psychiatric Association (EPA) guidance on prevention of mental disorders states "There is considerable evidence that various psychiatric conditions can be prevented through the implementation of effective evidence-based interventions' (Bolton 2008: 6). A 2011 UK Department of Health report on the economic case for mental health promotion and mental illness prevention found that "many interventions are outstandingly good value for money, low in cost and often become self-financing over time, saving public expenditure" (Campion, Bhui, Bhugra, 2012: 68-70). In 2016, the National Institute of Mental Health re-affirmed prevention as a research priority area.

Mental disorders traditionally have been viewed as distinct, episodic, and categorical conditions. This view has been challenged by evidence that many disorders are sequentially comorbid, recurrent/chronic, and exist on a continuum. Using the Dunedin Multidisciplinary 
Health and Development Study, they examined the structure of psychopathology, taking into account dimensionality, persistence, co-occurrence, and sequential comorbidity of mental disorders across 20 years, from adolescence to midlife. Psychiatric disorders were initially explained by three higher-order factors (Internalizing, Externalizing, and Thought Disorder) but explained even better with one General Psychopathology dimension (Stein, Phillips, Bolton, Fulford, Sadler, Kendler, 2010).

There are many definitions and causes of mental retardation. The contemporary look at mental retardation is multilateral. It is not based only on cognitive or intellectual disorders. It also refers to the emotional and volitional (action) sphere, based on a holistic vision of a human being. In this article the abilities and limitations of mentally handicapped children are presented by defining the notion of mental retardation. The likely course and outcome of mental disorders varies and is dependent on numerous factors related to the disorder itself, the individual as a whole, and the social environment. Some disorders may last a brief period of time, while others may be long term in nature.

The article is focused on different classifications of this disease and characteristics of mental disabilities in particular, especially mild. All disorders can have a varied course. Longterm international studies of schizophrenia have found that over a half of individuals recover in terms of symptoms, and around a fifth to a third in terms of symptoms and functioning, with many requiring no medication. While some have serious difficulties and support needs for many years, "late" recovery is still plausible.

\section{Mental Retardation: Definition and Genesis}

Intellectual disability or in other terms mental retardation is a variously defined concept. It is generally accepted that "it is a state of reduced mental ability in relation to the normal state, characterized by underdevelopment or disturbances of perceptual processes, attention, memory and thinking as a result of pathological processes. Some definitions (...) also include disruptions in social adaptation" (Stein, Phillips, Bolton, Fulford, Sadler, Kendler, 2010).

According to The Great Universal Encyclopedia PWN intellectual disability is "a state of insufficient intellectual performance due to underdevelopment or damage to early cerebral tissue in childhood".

The WHO recognizes a significant decline in the overall level of intellectual functioning and difficulties in adaptive behavior occurring before the age of 18 , where incurable cases are known. A. and M. Clark noticed that intellectual disability is "the functioning of the below-average intellect, which arises during the developmental period and is accompanied by a decrease in the ability to adapt" (Clark, Watson, Reynolds, 1995).

A relatively broad definition of intellectual disability was proposed by Edgar Doll, a specialist in the field of mental retardation research. He characterized the intellectual disability as: "the state of social immaturity arose in the period of development resulting from the inhibition of the development of the intelligentsia of constitutional origin. This condition is indelible" (Caron, Rutter, 1991). The author distinguished the following six aspects of disability: social immaturity, development delay, inhibition of permanent development, imperishable in the process of maturation, low mental performance, constitutionality (relating to the body of the organism), irreversibility.

It's interesting to know that the American Society for Research on Mental Invalidity (American Association of Mental Deficiency) in 1959 introduced the term "mental 
retardation". According to their point of view, mental disability can be considered as "lower than average, the overall level of intellectual functioning that arose during the developmental period. It is accompanied by disorders in the areas of maturation, learning and social adaptation " (Clark, Watson, Reynolds, 1995). When describing the essence of this mental disease they define three components in it:

intellectual functioning (a great emphasis is placed on intellectual limitations of people with intellectual disabilities, which are determined on the basis of a clinical trial or intelligence test results);

preservation behavior which is understood as a set of social, conceptual and practical skills that need to be learned in order to be able to function normally in everyday life. Both people with and without intellectual disability can face various difficulties in everyday life. Practical skills include activities such as eating, drinking, dressing, preparing food, cleaning, moving around, using home appliances, taking medicine, or managing money. Social skills are primarily the observance of rules, responsibility, the ability to self-evaluate and demonstrate self-control. Conceptual skills are learning speech, reading and writing, etc.

support systems ( people with intellectual disabilities show the need for support and usually use different systems to implement it. They can be offered at various demand levels, such as: periodic, limited, extensive and ubiquitous. Some people with mental disability need support only in one area, but it often happens that it is needed in different ones.

Intellectual disability is very often defined on the basis of the limitations people who are affected. For example, some scholars propose defining intellectual disability as "significantly lower than the average general level of intellectual functioning (...), which is accompanied by significant limitations in the adaptive functioning, at least in the scope of two of many such skills, as: verbal communication, self-service, coping with household chores, interpersonal skills, use of social security funds, managing yourself, school skills, job, ways of spending free time, care for health" (Copeland, Shanahan, Costello, Angold, 2011: 252261).

This definition thus puts emphasis on the fundamental limitations and deficits faced by people with intellectual disabilities. A different aspect of intellectual disability, focusing on the psychological peculiarities of this phenomenon has been worked out recently. It is therefore "(...) a state in which the mind did not achieve normal development. (...) inadequate social adaptation, reduced learning ability and slow durability of puberty" (Clark, Watson, Reynolds, 1995). Psychologically, intellectual disability is expressed in socially immature behaviors in the environment surrounding the disabled person. Inadequate social and scientific skills, improper performance of social roles have an unintentional effect on reducing the maturation threshold at which a given person should be.

A Polish educator Maria Grzegorzewska (Grzegorzewska, 1985) considers intellectual disability to be of two kinds: oligophrenia, or mental underdevelopment from the birth of a child or the earliest childhood, where there is always a cessation of the brain's development of higher nervous functions; dementia occurring later as weakness, breakdown of cortical processes, damage to the mental functions of the current full-blown.

It is also worth noting that M. Grzegorzewska describes the developmental characteristics of a disabled person, at the same time establishes a mental retardation as a superior category for oligophrenia and dementia, which she understands as "mental retardation" (Grzegorzewska, 1985). On the other hand, she proposes, for the first time, a dynamic approach to intellectual disability as a feature that, although it determines in a certain 
way mental capabilities of the individual, does not prevent it from developing in any sphere of life.

Therefore, it can be concluded that Grzegorzewska sees a certain developmental potential in people with intellectual disability, which can be liberated through revalidation and allow them to achieve the maximum of their skills (Grzegorzewska, 1985).

The latest generally accepted and applied definition of intellectual disability since 1997 is included in the International Classification of Damage, Disability and Handicap (ICD - 10). It is referred to as "(...) inhibition or incomplete mental development, expressed primarily in the impairment of skills that manifest themselves in the developmental period and constitute a general level of intelligence, i.e. cognitive, speech, motor skills and social skills. May occur alone or with other mental and physical disorders " (Markon, 2010:273-288).

In theoretical resources about special education, four perspectives of intellectual disability's thinking are mentioned. At present, the holistic approach to disability is being underlined; hence each of these perspectives can explain what intellectual disability is (Stein, Dan, 2013: 656-662). So from a psycho-biological point of view intellectual disability is treated as a permanent condition, caused by specific organic damage, permanently damaging important structures in the brain. It is recognized that the adoption of a medical perspective excludes the legitimacy of the use of educational interactions, the applied interactions should be of a caring and healing nature.

Psycho - developmental perspective captures intellectual disability as a failure in human life. The development is determined by a large number of biological and extrabiological factors. The developmental approach implies the possibility of a holistic impact on the intellectually disabled person. Disability is treated not as an illness or an organic disorder, but as a psychological limitation, as a state to which a person comes as a result of an abnormal development process.

In the realm of psychosocial perspective intellectual disability is treated here as a process of entering the social role of the disabled, in accordance with social expectations.

Pedagogical point of view focuses primarily on limited or difficult learning.

There are many factors that cause mental retardation and they are very diverse. Development and its abnormalities are conditioned by exogenous (extrinsic) and endogenous (intrinsic) factors. Scientists and scholars use congenital and acquired nomenclatures. The group of inborn factors includes:

- hereditary:

- genetic causes are caused by abnormal genes transmitted by one (dominant disorder) or two parents (recessive disorders);

- chromosomal abnormalities, that is, their improper structure, as well as their incorrect number (e.g. Down Syndrome);

- diseases associated with poor metabolism (e.g. phenylketonuria);

- congenital:

- mother's infectious diseases in the first 3 months of pregnancy,

- chronic diseases of the mother (e.g. hypertension, kidney or liver disease),

- diseases caused by the mother during pregnancy,

- hormonal disorders of the mother during pregnancy,

- serological conflict (especially incompatibility of the RH group),

- unfavorable living conditions of the mother during pregnancy (biological, social).

The second group comprises the following exogenous factors: 
- $\quad$ during labor (mechanical birth injuries and asphyxia),

- after the birth of a child under 3 years of age (infectious diseases, skull injuries, various types of poisoning).

\section{Classification of Mental Disabilities in Modern Medicine}

The latest classification of intellectual disability is included in the International Classification of Disabilities and Handicap Disabilities (ICD-10) from 1997 and in the classification prepared by the American Psychiatric Association - Diagnostic and Statistical Manual of Mental Disorders (DSM IV) from 1994. In both these classifications, the IQ is quantitatively determined by the numerical value of the intelligence quotient (II or IQ) obtained by carrying out one or more standardized tests for intelligence.

There are four levels of mental disability in DSM-IV and ICD-10: mild, moderate, severe and profound:

- mild intellectual disability (MID)

Many people with this degree of disability may not be noticed. People with this mental disease show the intellectual level of adults characteristic of a twelve-year-old person. The characteristics of this level of disability are as follows: children start walking a bit later; school difficulties arise, which are however manageable. They work a bit slower than the rest of the group, have poorer vocabulary, and are more difficult to adapt to new situations. Many of them can be helped by using special educational programs. Usually, after graduation, they are independent and able to work, and some establish families. Such persons are, therefore, independent and socially resourceful, but they should not engage in professions requiring decisions, because they have not reached the stage of abstract thinking in cognitive development.

Mild degree of mental retardation according to the World Health Organization corresponds to a scale Wechsler II69-55 (standard II115-85), Binet scale II67-52 (standard II100-84).

\section{- moderate intellectual disability (MOID)}

People with such kind of intellectual disability have problems with establishing contact with the environment, their speech development is delayed, they cannot cope with school. In the pre-school period, they show difficulties in acquiring social rules (loyalty and cooperation), as well as physical awkwardness and slow motor development. They deal with self-service. They perform simple jobs, they can work in sheltered workshops. It is believed, however, that they should not set up families. People with this degree of intellectual disability show the intellectual functioning of an adult person of nine years old.

\section{- severe intellectual disability (SID)}

People with such degree of intellectual disability often require constant care, are experiencing difficulties in communicating with the environment and manifestations of aggression. These people show the psychological development of an adult of the age of six. Around 4-5 years old, they show a delay in psychophysical development. These people can master self-care, they can learn housework while under constant care, but they are not able to learn a profession. They can undertake jobs that do not require qualifications.

\section{- profound intellectual disability (PID)}

At this level there is a complete degradation of mental life. In extreme cases, deeply handicapped people do not go beyond the standard of vegetative life. It is noticeable that there are no cortical reactions, and that functioning is consistent with unconditional reactions, with 
even disorders at the level of these reactions, manifesting itself, for example, with the abolition or significant reduction in the sense of pain. People with profound intellectual disabilities are not capable of independent living and require constant care from the surroundings. Most often they require the care of specialized institutions.

In Europe the classification of mental retardation (adopted by the World Health Organization on January 1, 1968), based on a scale of 100 and the standard deviation 16, which divides the mental retardation into four levels.

1. Mild mental retardation falls between two and three standard deviations, taking the intelligence quotient from 52 to 67.

2. Moderate mental retardation defines the area between three and four standard deviations, distinguishing persons with IQ between 36 and 51 .

3. Severe mental retardation falls between four and five standard deviations, classifying people with an IQ between 20 and 35 .

4. Profound mental retardation is the area between five and more standard deviations, here include people with IQ from 0 to 19.

The classification of the Wechsler mental disability (also based on the IQ) is widely recognized, where the individual degrees of impairment are determined by the standard deviation.

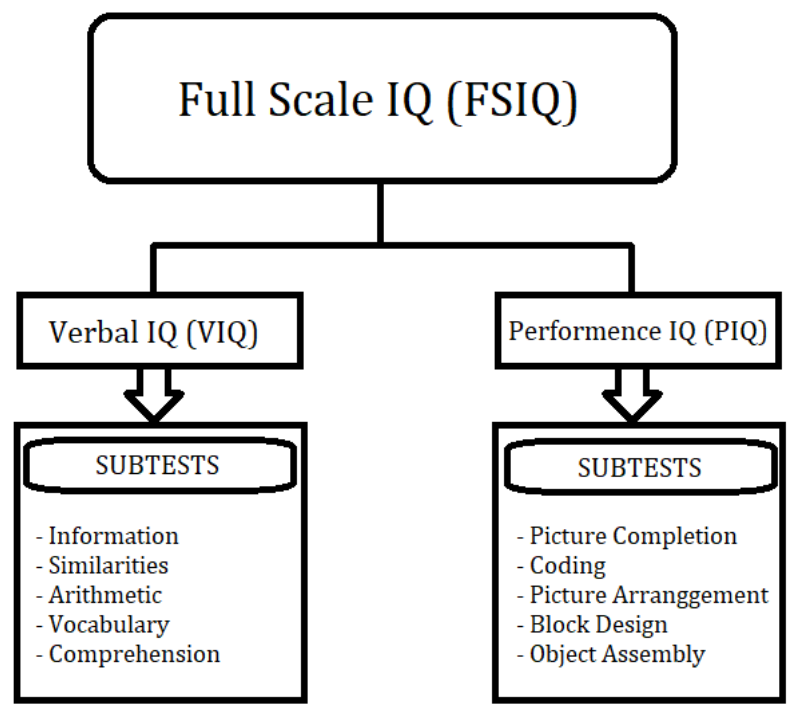

Fig. 1. Full Scale IQ (FSIQ)

At the first contact with children, who are mid-handed, you may not notice their intellectual defects. These units do not have physical signs of underdevelopment. Some of them have a rich vocabulary, the easiness of establishing contacts, expressing themselves to such an extent that during the conversation on the simplest topics concerning everyday life, we are unable to determine their underdevelopment. However, a student of a special school is characterized by disturbed orientation, cognitive, intellectual, emotional and motivational processes and difficulties in entering into social life. 
The most common school difficulties of mentally handicapped children are: disturbance of spatial orientation, poor level of graphomotor efficiency, more frequent motor development than in peers, poor concentration, common speech defects, slow work pace, problems in understanding the meaning of speech, problems of analysis and synthesis (visual, auditory, visual and auditory), the difficulty in recognizing letters and reading, difficulties in the sense of the text read, a significantly disturbed level of understanding of all rules, rules, definitions, difficult to create the concept of a number, limited level of memory performance of mathematical activities, lack of understanding of the content of text tasks and difficulties in solving them, disturbances of spatial imagination that make understanding and performing difficult geometric tasks, difficulties in orienting and using them, problems in linking new information with previously memorized ones, poor level of application of skills in practice, little independence in performing all tasks, often occurring psychomotor hyperactivity or inhibition, no criticism, selfishness, no anticipation of behavior , great impulsiveness, positivity to negative environmental influences, low level of motivation, small sensitivity to the needs of others, frequent manifestations of aggression (Copeland, Shanahan , Costello, Angold, 2011: 252-261).

All, above-mentioned difficulties do not have to be demonstrated in each case. Each student is different, in some sense unique (Kryński, Pluskota, 2012), his/her level of functioning at school, behavior, possibilities and limitations is individual

\section{Conclusions and suggestions}

In the population of people with intellectual disabilities, as much as $70 \%$ are individuals with mild disabilities. They differ from people in the intellectual norm, among other things, the reduced ability to orientate in a new, more complex situation. Recognition of such a degree of mental retardation is often a complex problem, because it results from similar symptoms of the behavior of a neglected and mentally handicapped child, because often the environmental conditions from which the child comes may affect its overall development.

The above description shows that a student of a special school with mild mental disability is characterized by a delayed mental development of almost all cognitive functions, resulting in visual and visual synthesis disorders, impaired auditory analysis and synthesis, spatial orientation disorders and emotional sphere disorders. Diversified speech defects, lack of understanding of many abstract terms, in particular, often affect mental disorders: complexes, depression, inhibitions, low resistance to conflict situations. These disorders reduce the ability to develop properly and build a full personality. The consequence of the abovementioned deficits in pupils is lack of general information, poor vocabulary, difficulties in correct speaking, reading and writing.

\section{References}

Bolton, D. (2008). What is Mental Disorder? An Essay in Philosophy, Science, and Values. OUP Oxford. P. 6. [in English].

Campion, J.; Bhui, K.; Bhugra, D. (2012). European Psychiatric Association (EPA) guidance on prevention of mental disorders. European Psychiatry, 27 (2), 68-80. DOI: 10.1016/j.eurpsy.2011.10.004. [in English]. 
Caron, C., Rutter, M. (1991). Comorbidity in child psychopathology: Concepts, issues and research strategies. Journal of Child Psychology and Psychiatry, 1063-1080. [in English].

Clark, L.A., Watson, D., Reynolds, S. (1995). Diagnosis and classification of psychopathology: Challenges to the current system and future directions. Annual Review of Psychology, 121-153. DOI: 10.1146/annurev.ps.46.020195.001005. [in English].

Copeland, W., Shanahan, L., Costello, E. J., Angold, A. (2011). Cumulative prevalence of psychiatric disorders by young adulthood: A prospective cohort analysis from the Great Smoky Mountains Study. Journal of the American Academy of Child and Adolescent Psychiatry, 252-261. DOI: 10.1016/j.jaac.2010.12.014. [in English].

Grzegorzewska, M. (1985). Pedagog $w$ stużbie dzieci niepetnosprawnych. Warszawa: Wydawnictwo WSPS. [in Polish].

Kryński, A., Pluskota, M. (2012). Pedagogika humanistyczna. Teoria $i$ praktyka. Częstochowa: Akademia Polonijna. [in Polish].

Stein, Dan J. (2013). What is a mental disorder? A perspective from cognitive-affective science. Canadian Journal of Psychiatry, 58 (12), 656-662. DOI: 10.1177/070674371305801202. [in English].

Stein, Dan J; Phillips, K. A; Bolton, D., Fulford, K.W. M., Sadler, J. Z., Kendler, K. S. (2010). What is a Mental/Psychiatric Disorder? From DSM-IV to DSM-V. Psychological Medicine, 40 (11),1759 - 1765. DOI:10.1017/S0033291709992261. [in English].

World Health Organization. (2004). Department of Mental Health and Substance Abuse; Prevention Research Centre of the Universities of Nijmegen and Maastricht Prevention of mental disorders: effective interventions and policy options: summary report (PDF). Geneva: World Health Organization. [in English]. 